\title{
Endometriosis and ovarian cancer risk, an epigenetic connection
}

\author{
Sarah Brunty ${ }^{1}$, Brenda Mitchell ${ }^{2}$, Nadim Bou-Zgheib $^{2}$, Nalini Santanam ${ }^{1 \wedge}$ \\ ${ }^{1}$ Department of Biomedical Sciences, Joan C. Edwards School of Medicine, Marshall University, Huntington, WV, USA; ${ }^{2}$ Department of Obstetrics \\ and Gynecology, Joan C. Edwards School of Medicine, Marshall University, Huntington, WV, USA \\ Contributions: (I) Conception and design: S Brunty, N Santanam; (II) Administrative support: None; (III) Provision of study materials or patients: \\ None; (IV) Collection and assembly of data: S Brunty, N Santanam; (V) Data analysis and interpretation: None; (VI) Manuscript writing: All authors; \\ (VII) Final approval of manuscript: All authors. \\ Correspondence to: Nalini Santanam, PhD, MPH. Department of Biomedical Sciences, $17003^{\text {rd }}$ Ave, 435 S BBSC, Joan C. Edwards School of \\ Medicine, Marshall University, Huntington, WV 25755, USA. Email: santanam@marshall.edu.
}

\begin{abstract}
Endometriosis is a gynecological disorder that affects 176 million women worldwide and 1 in 10 females in the United States. Endometriosis most often affects women of child-bearing age, with most going undiagnosed. Endometriosis also shares many characteristics common to invasive cancer and has been known to be associated with epithelial ovarian cancer. Ovarian cancer is the $11^{\text {th }}$ most common cancer among women and over 22,000 new cases will be diagnosed within the next year. Women most commonly diagnosed with this cancer are between the ages of 55-64 years, outside the range of the age of women affected with endometriosis. While no known cause of either disease has been established, epigenetic regulation is thought to play a major role in both. This review focuses on epigenetic changes that occur within each individual disease as well as those that are similar in both, suggesting a possible etiological link between the two diseases.
\end{abstract}

Keywords: Endometriosis-associated carcinoma; long non-coding RNA; epigenetic drugs

Submitted Mar 12, 2020. Accepted for publication May 18, 2020.

doi: $10.21037 /$ atm-20-2449

View this article at: http://dx.doi.org/10.21037/atm-20-2449

\section{Introduction}

Endometriosis is an elusive gynecological disorder defined by the growth and presence of endometrial tissue (lesions) outside of the uterus (1). This ectopic endometrial tissue responds to hormones and drugs in the same manner as its eutopic counterpart, and its continued growth is dependent on the hormone estrogen (2-4). Approximately $10-15 \%$ of women of child-bearing age suffer from endometriosis. Of the 1 in 10 women who suffer from it, $20-25 \%$ show no signs or symptoms, while others may experience symptoms such as infertility, chronic pelvic pain, or both (5-7). Currently, there are three main theories on how endometriosis originates (8). The most widely accepted is Sampson's theory, based on the retrograde shedding of endometrial cells into the abdominal cavity during menstruation, which in turn promotes a chronic inflammatory response (9). The inflammatory response in endometriosis has been studied extensively including in our laboratory (10-20). The second is the coelomic metaplasia theory in which the mesothelium differentiates in situ into endometrial tissue (21). The third is the embryonic origin theory in which the lesions are already present throughout the development and maturation in women (22). Other theories include stem cells (23), genetics, and autoimmune disease $(24,25)$. In addition to the lesions, affected women have an increased volume of peritoneal fluid (PF), that comprises of inflammatory cells, pain-inducing molecules, growth factors, cytokines/chemokines, etc. $(10,11,19,26)$. Our studies and others have shown a dynamic role for the $\mathrm{PF}$ in the etiology of endometriosis and the symptoms

$\wedge$ ORCID: 0000-0003-3343-6081 
associated with this disease $(10,11,19,27-29)$. While endometriotic lesions are not considered malignant, they do have cancer-like properties such as invasion of local organs, abnormal growth, and genetic damage $(30,31)$.

Ovarian cancer is diagnosed annually in nearly a quarter of a million women globally. It is responsible for 140,000 deaths each year making it the $4^{\text {th }}$ largest cause of cancer death in women and represents $1.3 \%$ of all new cancer cases. Epithelial ovarian cancer (EOC) contributes to $90 \%$ of the diagnosis. There are currently nine different types of EOC: high-grade serous, low-grade serous, mucinous, ovarian endometrioid invasive, clear-cell, undifferentiated or unclassified, primary peritoneal, Brenner tumors, and borderline tumors. With EOC, the early stages show no symptoms and there are no screening processes currently available. If diagnosed in the later stages, the cancer has usually spread to the peritoneal cavity and surgery is typically no longer an option. The 5-year survival rate for late stage diagnosis is $25 \%$ versus $90 \%$ for early stage (32).

Both endometriosis and ovarian cancer have many etiological similarities. One glaring commonality between the two is that there is no definitive known cause for either of these diseases. Epidemiological studies have shown that the prevalence of ovarian cancer in women with endometriosis is higher than sporadic ovarian cancer. Among the EOC subtypes, clear-cell, endometrioid invasive ovarian cancer, and low-grade serous are the most commonly seen in women with endometriosis $(33,34)$. A pooled analysis of 13 case-control studies of ovarian cancer in women with self-reported endometriosis consisting of 13,226 controls and 7,911 women with invasive ovarian cancer showed that endometriosis was associated with an increased risk of clear-cell with an odds ratio (OR) of 3.05, low-grade serous with an OR of 2.11, and endometrioid invasive ovarian cancer with an OR of 2.04 (35). In another study, $2.9 \%$ of women with endometriosis went on to develop ovarian cancer and the rate increased in women who had a longer history of endometriosis $(36,37)$.

These two diseases also share similar etiologies such as: damaged ovarian epithelium, elevated gonadotropins, androgen excess with progesterone deficiency, retrograde menstruation, oxidative stress and chronic inflammation (38). Recently there are several supporting evidence for the role of epigenetics in each of these diseases and possibly linking them. Figure 1 summarizes some of the commonalities that are seen in both endometriosis and ovarian cancer. In this review, we will only highlight the epigenetic mechanisms that they share. Some of these mechanisms have been linked to the transformation of endometriosis to its associated carcinomas, however others have been shown to be expressed in each of these diseases independently.

\section{Epigenetic mechanisms}

Epigenetics refers to the stable inheritance of phenotypes of cells and organisms without changes in DNA sequence or DNA content. This could involve DNA methylation, histone modifications, or changes in microRNAs (Figure 2). The changes and modifications that occur are reversible and dynamic and can be modified by environmental or lifestyle factors throughout an organism's entire lifespan $(39,40)$.

The most common epigenetic mechanism which regulates many cellular processes, including embryonic development, transcription, chromatin structure and stability (41) is DNA methylation. This involves the covalent transfer of a methyl group to the $\mathrm{C}-5$ position of the cytosine ring of DNA by DNA methyltransferases (DNMTs) (42). The donor molecule then dissociates, and the methylated DNA is released. DNMTs initiate and maintain the DNA methylation patterns, which are then regulated by methyl-CpG binding proteins (MDBs) that recognize and read the methylation marks created by the DNMTs (41). Typically, when a CpG island becomes methylated in cancer cells, loss of gene expression or silencing occurs due to the methylation of the islands inhibiting specific transcription factors from binding, as well as the recruitment of MDBs and their repressive chromatin remodeling functions (41). The methylation of CpGs is irreversible and can be passed down to daughter cells $(43,44)$.

The second common type of epigenetic change is histone modifications. These modifications are commonly referred to as post-translational modifications. They occur on the $\mathrm{N}$-terminal histone tails that extend from the nucleosomes, which then affects the chromatin structure, and the recruitment of remodeling enzymes. One of the widely investigated histone modification is histone methylation, which occurs mainly on lysines and arginines that are present on the histone tails, but does not change the charge of the tail itself. These changes can be reversed by demethylation by lysine demethylase 1 (LSD1). The second most common modification is histone acetylation which occurs on the lysines of the tails and is regulated by histone acetyltransferases (HATs) and histone deacetylases (HDACs). Acetylation of lysine in the histones results in removal of its positive charge thus weakening its interaction with the DNA. The third modification is histone 


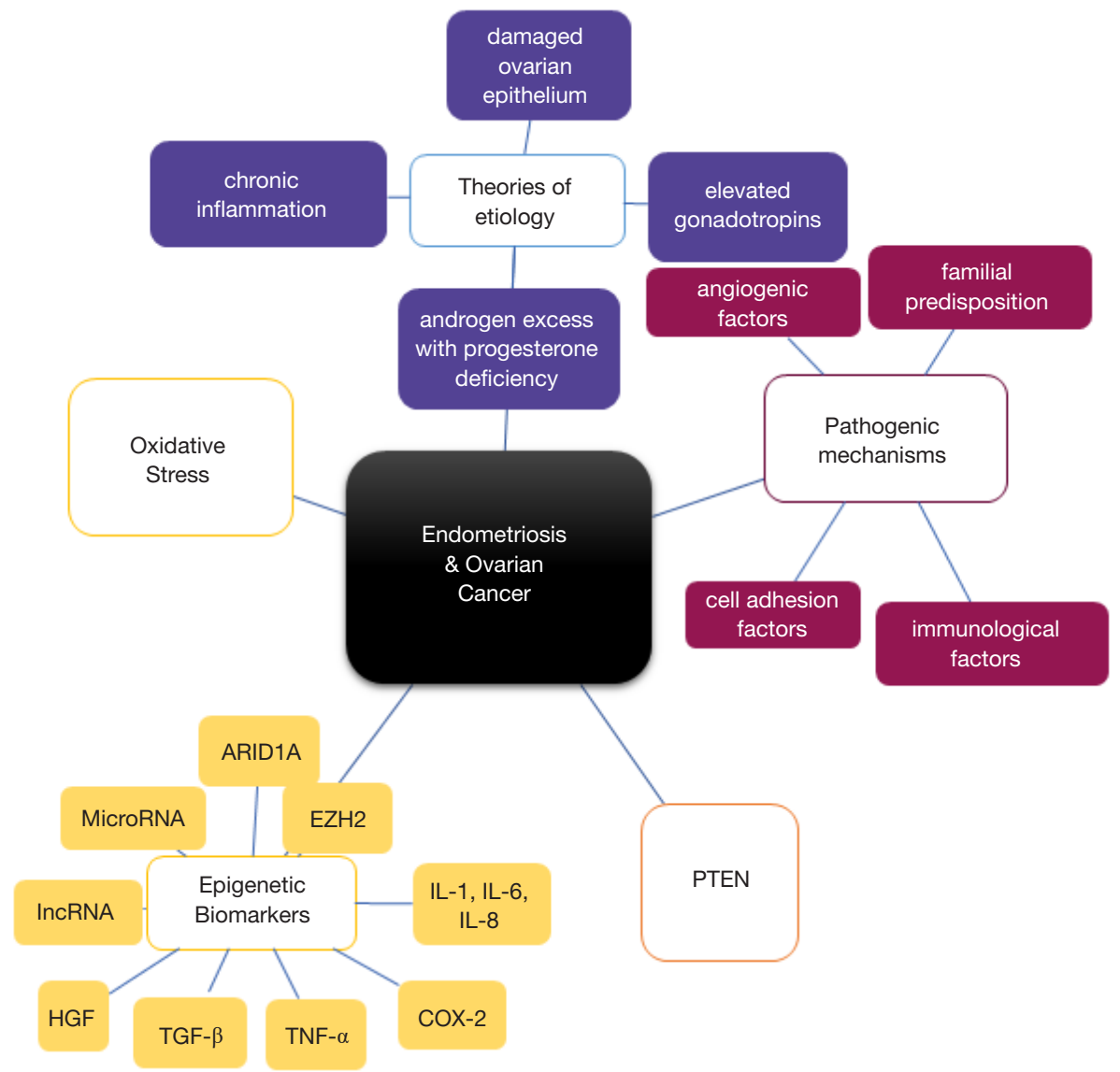

Figure 1 Etiological pathways that are commonly shared between endometriosis and ovarian cancer.

phosphorylation of serines, threonines and tyrosines, which adds a negative charge and changes the conformation of the histone on the nucleosome structure $(45,46)$. Chromatin remodeling has been shown to have a higher level of complexity and diversity than DNA methylation. The dynamic changes occurring affect the chromatin structure which influences the gene expression. Gene expression can also be affected by chemical modifications of histone proteins themselves (47).

One of the key regulatory complexes involved in histone modifications is the Polycomb repressive complex 2 (PRC2). A major subunit of the PRC2 complex is Enhancer of Zeste Homologue (EZH2), a histone methyltransferase (48-50). EZH2 plays a key role in transcriptional repression through chromatin remodeling, thus regulating gene expression as well as maintaining cell identity and oncogenesis (51). EZH2 activates $\mathrm{H} 3 \mathrm{~K} 27$ through trimethylation, H3K27me3 (52). This trimethylation causes gene silencing in cells. Studies have shown elevated EZH2 expression levels in various cancers $(49,53,54)$.

MicroRNAs (miRNAs) are an emerging third field of study in epigenetics which play a role in regulating gene expression. miRNAs are single-stranded non-coding RNAs, usually consisting of 22 nucleotides, that perform gene regulation without changing the DNA sequence. MiRNAs function in RNA silencing by two mechanisms. The first is via mRNA degradation where there is complete complementarity between the miRNA and the target mRNA nucleotide sequences. A single miRNA may be complementary to one or more mRNA, meaning that the gene silencing is not very specific (55-57). The second is by preventing the mRNA from being transcribed if the miRNA sequence is not completely complimentary. Several miRNAs control the expression of important epigenetic regulators which include DNMTs, HDACs and PRC2 complex, thus forming an epigenetic-miRNA regulatory circuit and feedback network $(58,59)$.

Epigenetics and endometriosis 


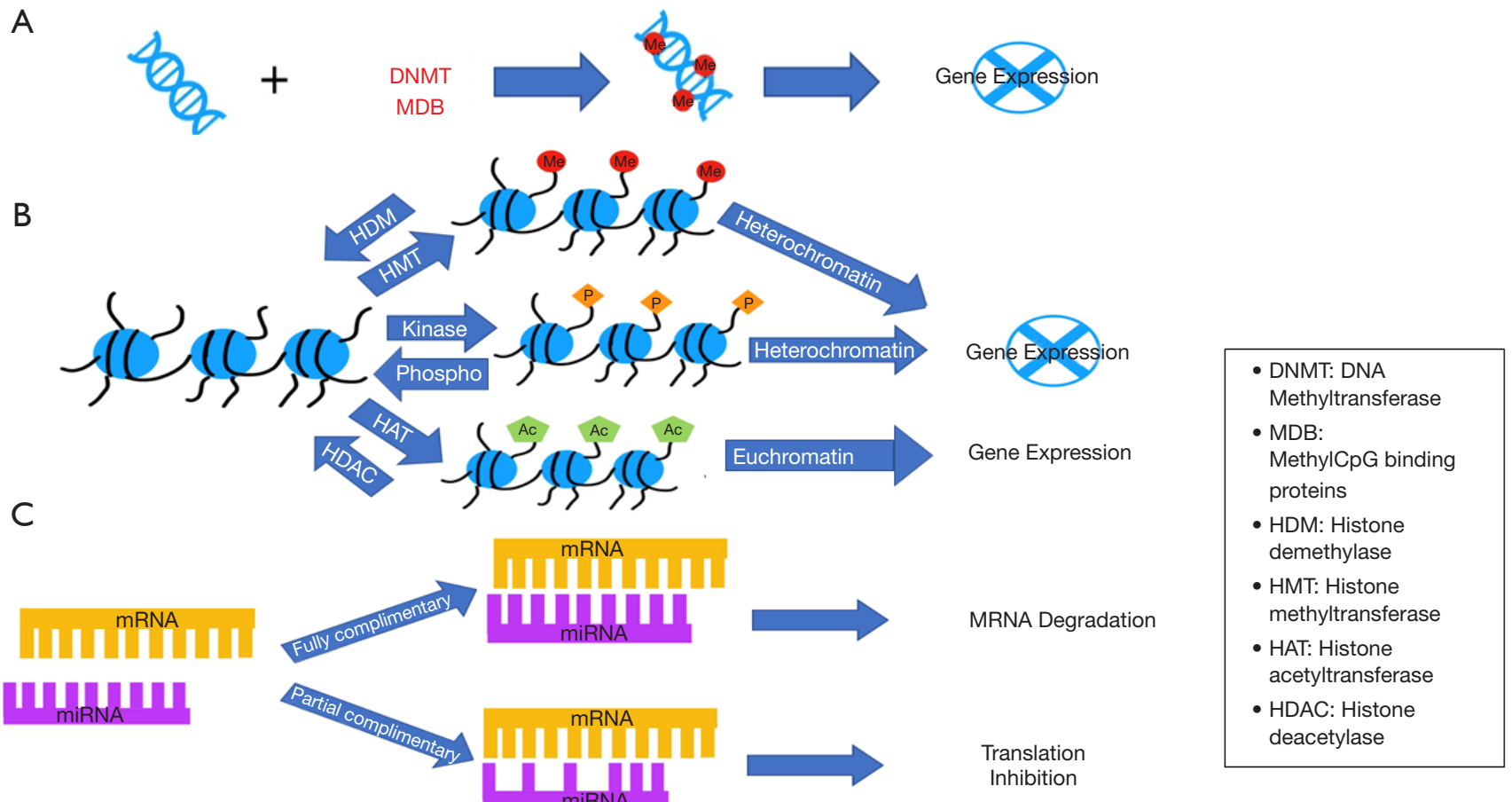

Figure 2 The three types of epigenetic mechanisms: (A) DNA methylation; (B) Histone modifications; (C) microRNA.

With no well-defined etiology known for endometriosis, epigenetics may be involved in its pathogenesis. Figure $3 \mathrm{~A}$ summarizes some of the recently reported studies that supports the role of the three epigenetic processes in endometriosis. There are evidences that show an over expression of the three DNMTs (DNMT 1, 3A, and 3B) in ectopic compared to eutopic tissues in women with endometriosis (60). Endometriosis is steroid responsive disease. The increase in ER $\beta$ mRNA levels in endometriotic tissues when compared to control tissue is due to promoter hypomethylation (61). Studies have similarly shown hypermethylation of the PR-B promoter region in endometriosis leading to its downregulation (62). Steroidogenic factor-1, SF-1, a nuclear receptor that regulates many genes in the hypothalamic-pituitary-adrenal endocrine axis, is expressed in ectopic endometriotic tissue but not eutopic tissue (63). Its aberrant expression in endometriosis is due to its hypomethylation in the CpG islands. SF-1 is also recruited to the promoter of steroidogenic genes and is a key regulator for estradiol synthesis and essential for growth and inflammation of endometriotic tissue. In endometriosis, E-cadherin, involved in cell-cell adhesion, is down-regulated and hypermethylated in its promoter region (64). Its expression can be induced by treatment with a histone deacetylase. The hypermethylation of Homeobox A10, HOXA10, a highly conserved homeobox gene, results in its reduced expression in the eutopic endometrium in women with endometriosis, resulting in defective uterine receptivity $(39,65)$. Decreased expression of HOXA10 results in the activation of autophagy in endometriosis (66).

In addition to the various enzymes involved in histone modifications (HDAC, HATs, HMTs and MeCP2) (39), studies from our laboratory provided the first evidence of an increased expression of EZH2/H3K27me3 in endometriotic lesions (67) which was later confirmed by other groups (68). EZH2 also enhances epithelial-mesenchymal transition in endometriosis (69). In endometriosis, the levels of H3K9 and H4K16 acetylation were lower, but methylation levels of $\mathrm{H} 3 \mathrm{~K} 4$ and $\mathrm{H} 3 \mathrm{~K} 9$ were higher (70).

The expression and function of miRNAs, including their role in epigenetic regulation, are an emerging area of focus in endometriosis (71). Many mRNA transcripts are differentially regulated in endometriotic lesions when compared to those of eutopic tissues. There are several studies that have determined circulating as well as tissue specific miRNA profiles in endometriosis. One such 


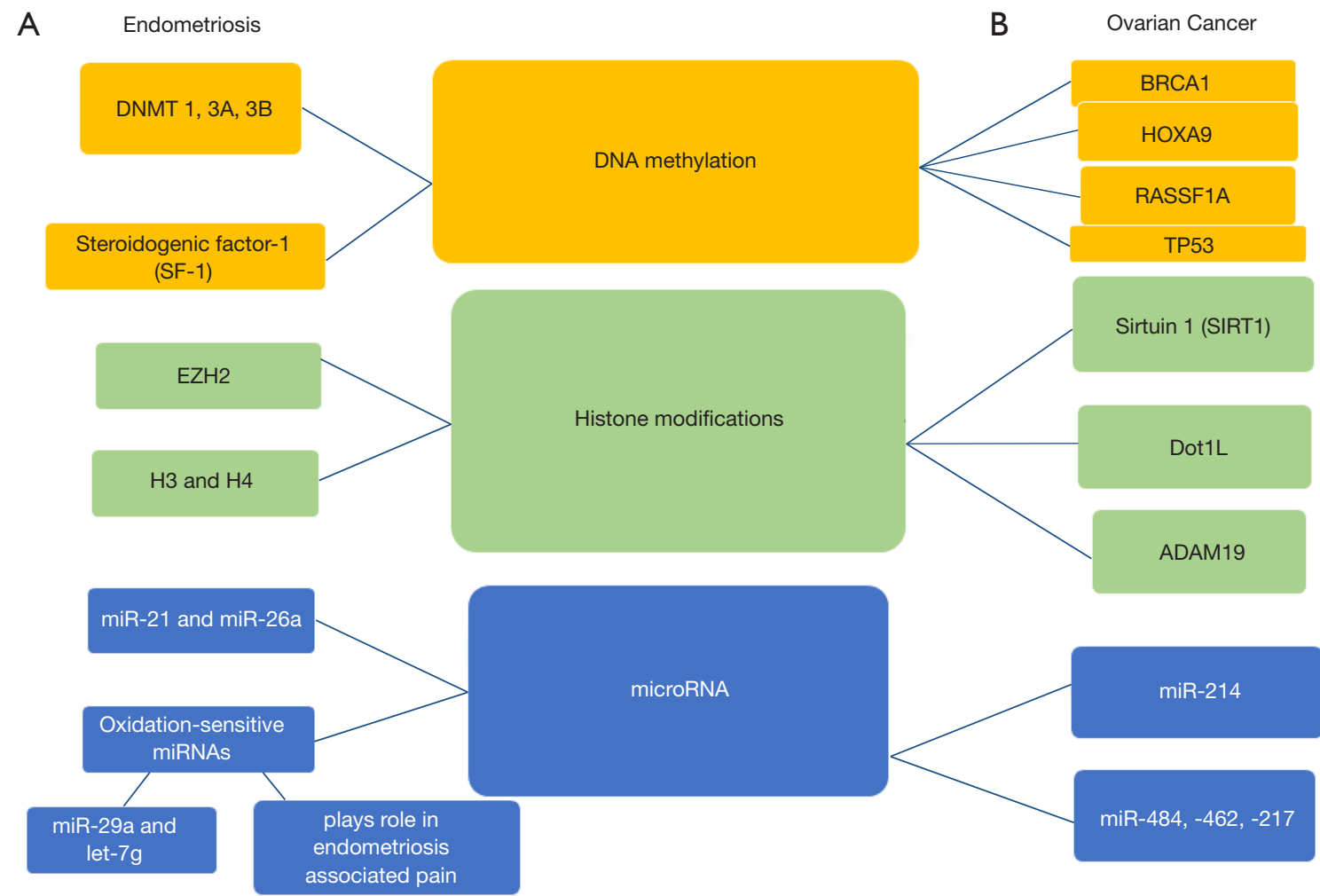

Figure 3 List of epigenetic marks that have been identified and discussed in the review: (A) endometriosis and (B) ovarian cancer.

study identified differential expression of 48 miRNAs in a microarray analysis, with the majority of these miRNAs displaying a reduced expression in eutopic and ectopic tissue when compared with control tissue. Validation by qRT-PCR demonstrated dysregulation of 2 specific miRNAs, miR-21 and miR-26a (72). Our laboratory recently showed that oxidation-sensitive miRNAs such as miR-29a and let-7g may play a role in endometriosis associated pain (11). A recent study identified miR-141 by driving the TGF $\beta 1 /$ SMAD2 signaling activates epithelial-to mesenchymal transition (EMT) in endometriosis (73).

\section{Epigenetics and ovarian cancer}

DNA methylation is also an important epigenetic biomarker for EOC (74). Figure $3 B$ summarizes some but not all of the epigenetic changes observed in EOC. BRCA1's promoter methylation is shown to be connected to malignancy in ovarian cancer (75). Higher silencing of this gene is observed in high-grade serous ovarian cancer (HGSOC) and is also connected with lower overall survival of patients. The HOXA9 promoter is hypermethylated in early-stage tumors of EOC (76). Comparing HOXA9 promoter methylation in normal endometrium from women with EOC and healthy controls, it was determined that this methylation is predicative of presence of EOC and the more methylation present, the more progressive was the cancer (77). The RASSF1A gene is a tumor suppressor gene, which is often repressed in ovarian cancer due to DNA methylation (78). RASSF1A is also a direct target of $\mathrm{H} 3 \mathrm{~K} 27 \mathrm{me} 3$ which results in its downregulation in ovarian cancer cells (79). The most commonly mutated gene in HGSOC according to Cancer Genome Atlas is TP53, which is involved in signaling pathways that halt cell cycle progression or induce apoptosis. It is an important tumor suppressor gene and the methylation of its promoter is seen in EOC (80). Sirtuins, also referred to as SIRTs, are NAD-dependent histone deacetylase that function as both an oncogene and a tumor suppressor, as well as maintains self-renewal and pluripotency of embryonic stem cells. In ovarian cancer, SIRT1 expression, as well as its deacetylase activity, was higher when compared to ovarian surface epithelium. This increased SIRT1 expression resulted in chemoresistance in ovarian cancer as well as increased the 
aggressiveness of the disease (81). DOT1-like protein, Dot1L, is the only known methyltransferase for H3K79 which is involved in cell cycle regulation and DNA repair. Dot1L expression was lost in ovarian cancer cells resulting in the promotion of cell invasion and induction of stem-cell like properties. This loss also downregulated E-cadherin and Tight Junction Protein 1, promoting EMT but upregulated the expression of Aldehyde Dehydrogenase 1 Family Member A1, by Wnt signaling activation (82). A Disintegrin and Metalloproteinase 19, ADAM19, is part of the ADAM family and has been shown to be involved in cell-cell and cell-matrix interactions as well as being an active metalloproteinase involved in cell migration and signal transduction. ADAM19 is also a target of TGF- $\beta 1$, whose pathway is often dysregulated in ovarian cancer. In ovarian cancer cells that were refractory to TGF- $\beta 1$ stimulation, ADAM19 expression was lower. These cells with disrupted TGF- $\beta 1$ signaling also had repressive histone marks, $\mathrm{H} 3 \mathrm{~K} 27 \mathrm{me} 3$ and $\mathrm{H} 3 \mathrm{~K} 9 \mathrm{me} 2$, associated with the promoter region of ADAM19 causing epigenetic dysregulation (83).

miR-214 is a miRNA that is involved in pro-growth and anti-apoptotic pathways and is a target in EOC. Its deregulation, leads to survival and resistance of the cell. This miRNA targets phosphatase and tensin homolog (PTEN), a tumor suppressor, which leads to activation of the Akt pathway, and is associated with cell survival (84). Other miRNAs that were studied in ovarian cancer are miR-484, -642 and -217, which predict chemoresistance. When secreted by ovarian cancer cells, these miRNA targets the VEGF signaling pathway which regulates vascular development and angiogenesis by acting directly on the VEGFB protein or inhibiting the VEGFR2 receptor in the endothelial cells (85).

\section{Common epigenetic marks in endometriosis and ovarian cancer}

\section{Epigenetic markers}

\section{ARID1A}

The AT-Rich Interaction Domain 1A (ARID1A) gene encodes a member of the SWI/SNF family and is required for transcriptional activation of genes that are normally repressed by chromatin (86). Mutations in ARID1A result in the loss of BRG-associated factor 250a (BAF250a), a protein with an important role in cell proliferation and tumor suppression, and a possible link between endometriosis and endometriosis-associated carcinomas. Samartzis et al. observed a complete loss of BAF250a in benign endometriotic lesions and that the expression of BAF250a was lower in ovarian endometriosis when compared to eutopic endometrium, peritoneal endometriosis, and deepinfiltrating endometriosis (87). The expression of this gene was also examined using whole transcriptomics on samples obtained from the Ovarian Cancer Research frozen-tumor bank. Mutations in ARID1A were present in $46 \%$ of ovarian clear-cell carcinomas and $30 \%$ of endometroid carcinomas, but none were seen in any of the HGSOC samples examined. These mutations also correlated with a loss of BAF250a expression in the tumors. Since these mutations were seen in the preneoplastic lesions, it could indicate an earlier event in the transformation from endometriosis to ovarian cancer (88).

\section{RASSF2}

Ras Association Domain Family Member 2, RASSF2, is highly expressed in humans and is a known tumor suppressor gene whose down-regulation in cells is due to promoter methylation. One study showed that RASSF2 may be a candidate gene for the malignant transformation of ovarian endometriosis. In this study, researchers used fresh tissue samples from endometriosisassociated ovarian carcinoma patients and found RASSF2's promoter was hypermethylated and the protein expression was significantly lower in the neoplastic tissue when compared to the ectopic endometrial tissue, suggesting that this epigenetic inactivation is an early event in the transformation (89).

\section{PTEN}

PTEN is another genetic alteration seen in ovarian cancer and endometriotic lesions suggesting a possible malignant transition. Using a mouse model, Dinulescu et al. induced endometrioid ovarian cancer and saw that when PTEN was deleted in the background of oncogenic $K$-ras activation, endometriotic precursor lesions were formed. These lesions went on to develop invasive endometrioid ovarian carcinoma (90). In endometriosis, overexpression of PTEN significantly increased apoptosis as well as inhibited the cell cycle (91). Cells expressing low levels of PTEN showed increased angiogenesis and the VEGF expression was high (91). In ovarian cancer, PTEN can suppress its growth by negatively regulating the PI3K/Akt pathway and that an abnormal expression of this pathway is associated with poor prognosis (92). Also, as discussed earlier many of the miR- 
A

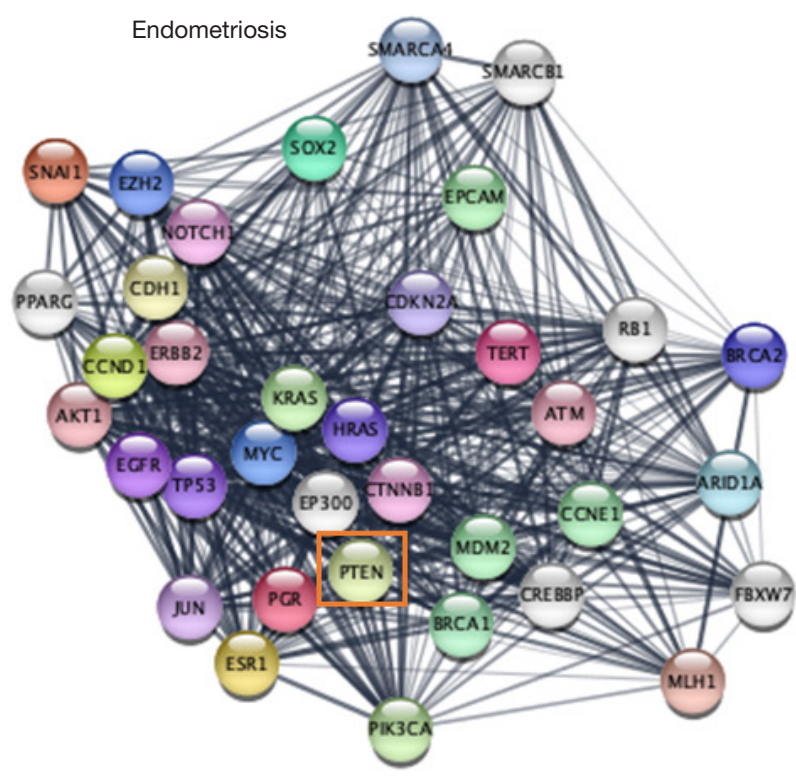

B

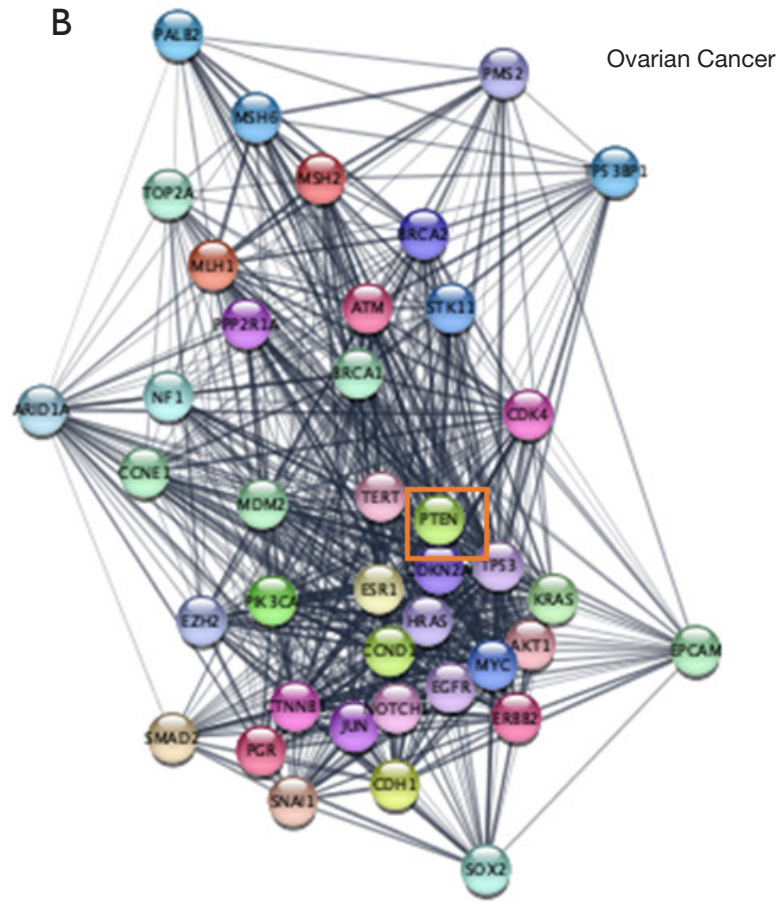

Figure 4 String network centered around PTEN developed using CytoScape. (A) Endometriosis and (B) ovarian cancer. Many of the PTEN network genes such as ARID1A and EZH2 are commonly regulated in both the diseases.

214 effects in ovarian cancer were mediated by targeting PTEN. One study using a mouse model examined using PTEN as a gene therapy in ovarian cancer and found that overexpression of PTEN resulted in lower tumor growth and an increased survival time (93). Figure 4, shows string networks centered on PTEN for both endometriosis and ovarian cancer.

\section{$\mathrm{EZH2}$}

$\mathrm{EZH} 2$, as described earlier, is shown to play a role in endometriosis by activating $\mathrm{H} 3 \mathrm{~K} 27 \mathrm{me} 3$ and shutting down transcription (94). EZH2 is also shown to play a role in ovarian cancer by enhancing angiogenesis $(54,86)$. Higher levels of EZH2 correlated to a worse prognosis for EOC patients (54). Interestingly, when miR-214 is downregulated in ovarian cancer, EZH2 is upregulated, increasing cell proliferation and transcriptional repression through $\mathrm{H} 3 \mathrm{~K} 27 \mathrm{me} 3$ in the same manner as that seen in endometriosis (95).

\section{microRNA}

There are several studies where differential expression of miRNAs have been studied in either endometriosis or ovarian cancer, however, Braicu et al. compared miRNAs in both these diseases in the same study. It was found that there were more differentially expressed miRNA in endometriosis compared to ovarian cancer and most were linked to EMT (96). Two common miRNAs overexpressed in both diseases were miR-325 and miR-492 (96). While the expression of miR-325 was upregulated in both diseases, it was more upregulated in ovarian cancer suggesting miR-325 as having a role in the transition from endometriosis to ovarian cancer (96). Not many studies have examined the levels of these miRNAs in either disease but upregulation of miR492 has been shown to decrease PTEN expression which increases the level of AKT activation in cancer cells (97). miR-325 is downregulated in hepatocellular carcinoma but its upregulation inhibited cell invasion and proliferation by targeting HMGB1 (98). miR-21 which was earlier discussed as potentially having a role in endometriosis (72), can cause a cisplatin resistance in ovarian cancer and that its inhibition can enhance the tumor suppressor Programmed Cell Death 4, PDCD4, as well as promote apoptosis (99).

\section{miR-199a}

In endometriosis, Wang et al. found that miR-199a relative expression levels were higher in samples from endometriosis 
patients versus controls and noted that circulating levels of miR-199 increased as the disease progressed. Interestingly, it was also noted that the concentration of miR-199a was significantly higher in ovarian endometriosis than in other types of endometriosis (100). Downregulation of miR-199a correlated with an upregulation of HIF $1 \alpha$ in ovarian tumors when compared to normal tissues and to shorter survival in patients. When miR-199a was increased it promoted cisplatin-induced apoptosis by suppressing HIF $1 \alpha$ in the cells (101).

\section{miR-135}

MiRNA-135 represses HOXA-10 thereby altering endometrial receptivity and implantation (102). In the endometrial lesions, expression of $m i R-135 a / b$ was decreased when compared to the normal tissue. However, expression was increased in the secretory phase compared to the proliferative phase, by over 10-fold in the lesions and nearly 8 -fold in the tissues, suggesting a role in endometrial function and implantation in women with endometriosis (103). In ovarian cancer, it was found that miR-135a was significantly down regulated in EOC tissues which correlated to a high expression of HOXA10 in these tissues when compared to controls. This low expression of miR-135a also correlated with a shorter progression-free survival. The downregulation of HOXA10 also resulted in changes in apoptosis-associated genes, such as caspase-3 and p53, which may control EOC tumor growth (104).

\section{miR-141}

miR-141 is a member of the miR-200 family known to modulate the Notch, p53, MAPK, and ErbB signaling pathways. In endometriosis, expression of this miRNA was decreased in the ectopic endometrium when compared to normal and eutopic endometrium as well as being decreased in the ectopic endometrial stromal cells. When cells were transfected with miR-141, a lower growth rate was observed, but it promoted cell proliferation and reduced their migration. Cells transfected with an miR-141 mimic decreased Kruppel like factor 12 (KLF-12), an oncogene seen in several cancers, suggesting that it is a direct target of miR-141 (105). In ovarian cancer cells, Mak et al. showed that miR-141 significantly increased their anchorageindependent growth. This upregulation also correlated to cancer metastasis. When miR-141 was inhibited in cells, a significant reduction in cell viability by over $50 \%$ was seen. miR-141 and KLF-12 were shown to have an inverse relationship in this disease as well, it had a tumor- suppressive function in ovarian cancer. Overall, their study showed that miR-141 suppresses KFL-12 which causes cells to escape apoptosis, survive, and metastasize (106). Wu et al. showed that this oncogenic miRNA was higher in ovarian cancer when compared to its concurrent endometriosis and could be a possible marker that distinguishes the two and could be a possible mediator of transformation between the diseases (107).

\section{Long non-coding $R N A$}

Long non-coding RNAs (lncRNA) are newer biomarkers implicated in various diseases including ovarian cancer (108) and endometriosis $(109,110)$. They are longer than 200 nucleotides and not translated into protein. Though long considered to be "junk", recent evidences support its role in several diseases (111). As with miRNAs, some of the lncRNA discussed below though shown to be playing a role in both these diseases, have not yet been directly linked to each other.

\section{MALAT1}

Metastasis-associated lung adenocarcinoma transcript 1 (MALAT1) is a lncRNA that plays a role in various types of cancers, including ovarian (112). Its function is mediated through PI3K/AKT signaling pathway and its silencing has resulted in a decrease in EMT and an increase in E-cadherin levels in some cancers (108). In endometriosis, there is a time-dependent increase in MALAT1 in the stromal cells treated with hypoxia correlating with an increase in HIF$1 \alpha$ in the cells. These elevations caused an activation of pro-survival autophagy pathway, reducing apoptosis in endometriosis (113). MALAT1 is overexpressed in ovarian cancer, with higher expression correlating to a larger tumor size and metastasis. When MALAT1 was knocked down in an ovarian cancer cell line, SK-OV-3, there was a decrease in cell viability as well as proliferation, migration, and invasion suggesting that MALAT1 plays a role in ovarian cancer growth and development (112).

\section{H19}

In normal cells, H19 is an imprinted lncRNA whose function is acting as a molecular sponge for miRNA let-7 and inhibiting its function. Endometrial H19 expression is regulated by estrogen and progesterone positively and negatively, respectively (114). Ghazal et al. showed that the expression of $H 19$ was significantly decreased in women with endometriosis when compared to normal women, 
which correlated with an increase in let-7 activity. The increase in this miRNA inhibited the post-transcriptional expression levels of the IGF1 receptor (IGF1R) and resulted in decreased proliferation of endometrial stromal cells (115). In ovarian cancer, H19 expression was shown to be increased in cisplatin-resistant cells and this expression had a strong correlation with cancer recurrence. When H19 was knocked down in cisplatin-resistant cells, resistance was reversed in cells that were more sensitive to hydrogenperoxide treatments (116).

\section{BANCR}

BRAF-activated non-coding RNA, BANCR, is increased in cancers such as thyroid, lung, and bladder (117). In an endometriosis rat model, the volume of the grafts in the BANCR inhibitor group was smaller than that of the control group as well as the development of the graft tissues being repressed prominently. Using $\mathrm{H} \& \mathrm{E}$ staining, it was shown that there was fewer interstitial cells and a declined blood supply when compared to the control group. BANCR can suppress production of angiogenic factors in endometriosis which could repress development of ectopic endometrial tissues (118). BANCR expression was examined in EOC patient tissues and shown to be increased when compared to normal tissues. This high expression also correlated with an advanced FIGO stage, high CA125 expression, and with an occurrence of metastasis in the lymph nodes (119).

\section{Drug therapy}

With the evidence accumulating that epigenetic changes may be the underlying cause for the initiation and progression of both diseases, many physicians and scientists have turned to targeting epigenetic pathways for treatment. In ovarian cancer, one of the most common epigenetic drugs tested is histone deacetyltransferase inhibitors, HDACi which work by increasing the level of acetylated histones, reactivating silenced tumor suppressor genes. Currently, only three HDACi, vorinostat, romidepsin, and panobinostat, have been approved by the FDA (120). These HADCi have very little effect alone, hence many are used in combination therapy. Treatments with DNMT inhibitors and HDACi have been investigated as a means of reversing platinum resistance in ovarian cancer. An HDACi that is currently in the preclinical stage for ovarian cancer is Trichostatin A, TSA. TSA inhibits class I and II HDACs as well as induces expression of P73 and facilitate Bax- dependent apoptosis in cancer cells that are resistant to cisplatin (121). One specific DNMT inhibitor, decitabine, was shown to lead to demethylation of many genes including those that were discussed in this review, such as HOXA10 and BRCA1 (122,123).

Hydralazine is an anti-hypertensive medication that was shown to inhibit non-nucleoside DNA methylation. This drug is in clinical trials along with valproic acid, VPA, which works through oxidative stress and generates reactive oxygen species to inhibit HDACs. This combination was tested in refractory solid tumors, including ovarian cancer. Out of 15 total patients with various solid tumors, four had partial response ( 3 ovarian cancer) and 8 (4 ovarian) had stable disease 4 weeks after completing one or two cycles of chemotherapy. Results showed that epigenetic drugs could be used to re-sensitize tumors to chemotherapy and should be used in combination (124). Many of the miRNA and lncRNA discussed above were also shown to play a role in chemotherapy resistance and could be future drug targets.

Bevacizumab (Avastin) is an antiangiogenetic drug used to target VEGF and inhibit proliferation and migration of the cells. Trials have shown that bevacizumab, when used as an initial therapy, improved progression free survival in ovarian cancer patients (125).

Another combination approach that is being examined is epigenetic inhibitors and immunotherapy, which has been used in cancers such as non-small cell lung cancer and melanoma with good success, however is in its very early stages of being used in ovarian cancer. Stone $e t$ al. found that in a mouse model of EOC, DNMTi and HDACi improved the response to immune checkpoint therapy. Specifically, the DNMTi 5-azacytidine, AZA, increased the number of $\mathrm{CD} 5^{+}$immune cells as well as the percentage of $\mathrm{NK}$ cells and active $\mathrm{CD}^{+}$cells in the microenvironment. This, in turn, reduced the tumor burden and extended the survival. A triple combination therapy consisting of DNMTi, HDACi, and an immune checkpoint inhibitor provided the greatest effects and longest overall survival (126).

In endometriosis, epigenetic drugs have not been explored to the same extent as that in ovarian cancer, but much of the research has focused on HMT inhibitors, specifically those that work against EZH2. The two common drugs of this type are 3-Deazaneplanocin A hydrochloride, DZNep, and GSK126. DZNep is a lysine methyltransferase EZH2 inhibitor and competitive s-adenosyl homocysteine hydrolase inhibitor, that blocks $\mathrm{H} 3 \mathrm{k} 27 \mathrm{me} 3$ and H4k20me3 (127). GSK126 is a selective, $\mathrm{S}$-adenosyl-methionine-competitive small molecule 
inhibitor of EZH2 methyltransferase activity and is more than 1,000-fold selective for EZH2 over other HMTs (128). In endometriosis, DZNep was shown to inhibit the growth of endometriotic lesions as well as attenuated EMT and reduced fibrosis in vivo (69). Although GSK126 is currently being investigated as a treatment for endometriosis, it has not been studied as extensively as DZNep (68). However, it has been studied in endometrial cancer (129). In ovarian cancer, GSK126 caused a regression of ARID1A-mutated ovarian clear cell carcinoma and decreased the number of tumor nodules in xenograft models (86). Recent studies showed that GSK126 suppressed the growth and improved the survival of Coactivator Associated Arginine Methyltransferase, CARM1-high expressing HGSOC and EOC as CARM1 promotes EZH2-mediated silencing of target tumor suppressor genes (130).

TSA is also being examined in endometriosis by looking at its effects on nonsteroidal anti-inflammatory drug activated gene I (NAG-1) expression and apoptosis. NAG1 , a member of the TGF- $\beta$ family and associated with apoptosis and cell growth is decreased in the endometrium of patients with endometriosis. Ectopic endometrial tissues when treated with varying concentrations of TSA resulted in induction of NAG-1 leading to apoptosis in a dosedependent manner (131). Other studies have also shown that TSA can reduce endometrial implants in mice (132). $\mathrm{Wu}$ et al. found VPA to induce cell cycle arrest, as well as p21 expression in endometrial stromal cells (133). Another group used a rat model of endometriosis to test the effects of VPA along with progesterone. They examined the serum levels of TNF- $\alpha$, lesion size, and body weight. Rats receiving VPA and/or progesterone showed a reduced lesion size and increased weight gain compared to the untreated rats as well as an improved response to thermal stimuli, suggesting that this combination may be an efficient treatment for endometriosis (134).

\section{Conclusions}

Throughout this review, epigenetic markers that have been identified and studied extensively in endometriosis, ovarian cancer, or both have been discussed. While neither disease has a definitive cause, there are many avenues that could be responsible to play a role in their etiology. And while endometriosis is not a disease that affects all women, and even in those who are affected, its phenotype is different, it is plausible to theorize that epigenetics plays a major role in its initiation and progression. Similarly, not much is known about how endometriosis transforms into carcinoma? Some of the common epigenetic markers found in these two diseases might play a plausible role in this linkage.

Many scientists around the world are focusing their efforts not only in identifying the cause of these diseases, but also on ways for its early detection and treatment. Many of the current treatments available are only of temporary relief, however with the gain of newer knowledge, better treatment options are growing for both these diseases. It is however getting clear from these studies that there is no single gene or marker that is truly responsible for either of these diseases, but however, a combination of multiple biomarker changes all working together is involved in the disease initiation and progression. What remains a fact is that more research is warranted in these two diseases, as more and more women are being diagnosed each year from them and hence need well designed diagnostics and therapeutics to curb them.

\section{Acknowledgments}

The authors thank Ms. Sandy White and Morgan Ruley for coordinating the clinical sample collection and Dr. David Jude, Chairman, Dept Ob-Gyn for his continued support. Funding: This work was partially supported by National Institutes of Health, P20GM103434 to the West Virginia IdeA Network for Biomedical Research Excellence (NS); Edwards Comprehensive Cancer Center Foundation grant (NS, NBZ, BM) and PhRMA Grant 218218 Pre-Doctoral Fellowship for Pharmacology/Toxicology (SB).

\section{Footnote}

Provenance and Peer Review: This article was commissioned by the Guest Editors (Stergios Boussios and Nicholas Pavlidis) for the series "Ovarian Cancer: State of the Art and Perspectives of Clinical Research" published in Annals of Translational Medicine. The article was sent for external peer review organized by the Guest Editors and the editorial office.

Conflicts of Interest: All authors have completed the ICMJE uniform disclosure form (available at http://dx.doi. org/10.21037/atm-20-2449). The series "Ovarian Cancer: State of the Art and Perspectives of Clinical Research" was commissioned by the editorial office without any funding or sponsorship. The authors have no other conflicts of interest to declare. 
Ethical Statement: The authors are accountable for all aspects of the work in ensuring that questions related to the accuracy or integrity of any part of the work are appropriately investigated and resolved.

Open Access Statement: This is an Open Access article distributed in accordance with the Creative Commons Attribution-NonCommercial-NoDerivs 4.0 International License (CC BY-NC-ND 4.0), which permits the noncommercial replication and distribution of the article with the strict proviso that no changes or edits are made and the original work is properly cited (including links to both the formal publication through the relevant DOI and the license). See: https://creativecommons.org/licenses/by-nc-nd/4.0/.

\section{References}

1. Burney RO, Giudice LC. Pathogenesis and pathophysiology of endometriosis. Fertil Steril 2012;98:511-9.

2. Gao $Y$, Shen $M, M a X$, et al. Seven Hormonal Biomarkers for Diagnosing Endometriosis: Meta-Analysis and Adjusted Indirect Comparison of Diagnostic Test Accuracy. J Minim Invasive Gynecol 2019;26:1026-1035.e4.

3. Yilmaz BD, Bulun SE. Endometriosis and nuclear receptors. Hum Reprod Update Hum Reprod Update 2019;25:473-85.

4. Curtis L, Adams HP. Endometriosis: From Identification to Management Clinician Reviews 2017;27:28-32.

5. Bulletti C, Coccia ME, Battistoni S, et al. Endometriosis and infertility. J Assist Reprod Genet 2010;27:441-7.

6. Fraser IS. Mysteries of endometriosis pain: Chien-Tien Hsu Memorial Lecture 2009. J Obstet Gynaecol Res 2010;36:1-10.

7. Coxon L, Horne AW, Vincent K. Pathophysiology of endometriosis-associated pain: A review of pelvic and central nervous system mechanisms. Best Pract Res Clin Obstet Gynaecol 2018;51:53-67.

8. Vinatier D, Orazi G, Cosson M, et al. Theories of endometriosis. Eur J Obstet Gynecol Reprod Biol 2001;96:21-34.

9. Sampson JA. Peritoneal endometriosis due to menstrual dissemination of endometrial tissue into the peritoneal cavity. Am J Obstet Gynecol 1927;14:422-69.

10. Ray K, Fahrmann J, Mitchell B, et al. Oxidation-sensitive nociception involved in endometriosis-associated pain. Pain 2015;156:528-39.

11. Wright KR, Mitchell B, Santanam N. Redox regulation of microRNAs in endometriosis-associated pain. Redox Biology 2017;12:956-66.

12. Symons LK, Miller JE, Kay VR, et al. The Immunopathophysiology of Endometriosis. Trends Mol Med 2018;24:748-62.

13. Luckow Invitti A, Schor E, Martins Parreira R, et al. Inflammatory cytokine profile of cocultivated primary cells from the endometrium of women with and without endometriosis. Mol Med Rep 2018;18:1287-96.

14. Donnez J, Binda MM, Donnez O, et al. Oxidative stress in the pelvic cavity and its role in the pathogenesis of endometriosis. Fertil Steril 2016;106:1011-7.

15. Murphy AA, Santanam N, Parthasarathy S. Endometriosis: a disease of oxidative stress? Semin Reprod Endocrinol 1998;16:263-73.

16. Santanam N, Murphy AA, Parthasarathy S. Macrophages, oxidation, and endometriosis. Ann N Y Acad Sci 2002;955:183-98; discussion 19-200, 396-406.

17. Santanam N, Song M, Rong R, et al. Atherosclerosis, oxidation and endometriosis. Free Radic Res 2002;36:1315-21.

18. Shanti A, Santanam N, Morales AJ, et al. Autoantibodies to markers of oxidative stress are elevated in women with endometriosis. Fertil Steril 1999;71:1115-8.

19. Santanam N, Kavtaradze N, Murphy A, et al. Antioxidant supplementation reduces endometriosis-related pelvic pain in humans. Transl Res 2013;161:189-95.

20. Akinwunmi BO, Babic A, Vitonis AF, et al. Chronic Medical Conditions and CA125 Levels among Women without Ovarian Cancer. Cancer Epidemiol Biomarkers Prev 2018;27:1483-90.

21. Matsuura K, Ohtake H, Katabuchi H, et al. Coelomic metaplasia theory of endometriosis: evidence from in vivo studies and an in vitro experimental model. Gynecol Obstet Invest 1999;47 Suppl 1:18-20; discussion 22.

22. Batt RE, Yeh J. Mullerianosis: four developmental (embryonic) mullerian diseases. Reprod Sci 2013;20:1030-7.

23. Maruyama T, Yoshimura Y. Stem cell theory for the pathogenesis of endometriosis. Front Biosci (Elite Ed) 2012;4:2754-63.

24. Ahn SH, Monsanto SP, Miller C, et al. Pathophysiology and Immune Dysfunction in Endometriosis. Biomed Res Int 2015;2015:795976.

25. Sasson IE, Taylor HS. Stem cells and the pathogenesis of endometriosis. Ann N Y Acad Sci 2008;1127:106-15.

26. Koninckx PR, Kennedy SH, Barlow DH. Pathogenesis of endometriosis: the role of peritoneal fluid. Gynecol Obstet 
Invest 1999;47 Suppl 1:23-33.

27. Ray KL, Mitchell BL, Santanam N. Power over Pain: A Brief Review of Current and Novel Interventions for Endometriosis-Associated Pain Journal of Endometriosis and Pelvic Pain Disorders 2014;6:163-73.

28. Bedaiwy MA, Falcone T. Peritoneal fluid environment in endometriosis. Clinicopathological implications. Minerva Ginecol 2003;55:333-45.

29. Bricou A, Batt RE, Chapron C. Peritoneal fluid flow influences anatomical distribution of endometriotic lesions: why Sampson seems to be right. Eur J Obstet Gynecol Reprod Biol 2008;138:127-34.

30. Kajiyama H, Suzuki S, Yoshihara M, et al. Endometriosis and cancer. Free Radic Biol Med 2019;133:186-92.

31. Thomsen LH, Schnack TH, Buchardi K, et al. Risk factors of epithelial ovarian carcinomas among women with endometriosis: a systematic review. Acta Obstet Gynecol Scand 2017;96:761-78.

32. Siegel RL, Miller KD, Jemal A. Cancer Statistics, 2017. CA Cancer J Clin 2017;67:7-30.

33. Robinson KA, Menias CO, Chen L, et al. Understanding malignant transformation of endometriosis: imaging features with pathologic correlation. Abdom Radiol (NY) 2020;45:1762-75.

34. Siufi Neto J, Kho RM, Siufi DF, et al. Cellular, histologic, and molecular changes associated with endometriosis and ovarian cancer. J Minim Invasive Gynecol 2014;21:55-63.

35. Pearce CL, Templeman C, Rossing MA, et al. Association between endometriosis and risk of histological subtypes of ovarian cancer: a pooled analysis of case-control studies. Lancet Oncol 2012;13:385-94.

36. Gandini S, Lazzeroni M, Peccatori FA, et al. The risk of extra-ovarian malignancies among women with endometriosis: A systematic literature review and metaanalysis. Crit Rev Oncol Hematol 2019;134:72-81.

37. Li J, Liu R, Tang S, et al. Impact of endometriosis on risk of ovarian, endometrial and cervical cancers: a metaanalysis. Arch Gynecol Obstet 2019;299:35-46.

38. Nezhat F, Apostol R, Mahmoud M, et al. Malignant transformation of endometriosis and its clinical significance. Fertil Steril 2014;102:342-4.

39. Guo SW. Epigenetics of endometriosis. Mol Hum Reprod 2009; 15:587-607.

40. Allis CD, Jenuwein T. The molecular hallmarks of epigenetic control. Nat Rev Genet 2016;17:487-500.

41. Robertson KD. DNA methylation and human disease. Nat Rev Genet 2005;6:597-610.

42. Jin B, Li Y, Robertson KD. DNA methylation: superior or subordinate in the epigenetic hierarchy? Genes Cancer 2011;2:607-17.

43. Sen A, Heredia N, Senut MC, et al. Multigenerational epigenetic inheritance in humans: DNA methylation changes associated with maternal exposure to lead can be transmitted to the grandchildren. Sci Rep 2015;5:14466.

44. Skinner MK, Guerrero-Bosagna C. Role of CpG deserts in the epigenetic transgenerational inheritance of differential DNA methylation regions. BMC Genomics 2014;15:692.

45. Bannister AJ, Kouzarides T. Regulation of chromatin by histone modifications. Cell Res 2011;21:381-95.

46. Berger SL. The complex language of chromatin regulation during transcription. Nature 2007;447:407-12.

47. Dhall A, Chatterjee C. Chemical approaches to understand the language of histone modifications. ACS Chem Biol 2011;6:987-99.

48. Chan HL, Beckedorff F, Zhang Y, et al. Polycomb complexes associate with enhancers and promote oncogenic transcriptional programs in cancer through multiple mechanisms. Nat Commun 2018;9:3377.

49. Simon JA, Lange CA. Roles of the EZH2 histone methyltransferase in cancer epigenetics. Mutat Res 2008;647:21-9.

50. Kim J, Lee Y, Lu X, et al. Polycomb- and MethylationIndependent Roles of EZH2 as a Transcription Activator. Cell Rep 2018;25:2808-2820.e4.

51. Kadoch C, Copeland RA, Keilhack H. PRC2 and SWI/ SNF Chromatin Remodeling Complexes in Health and Disease. Biochemistry 2016;5 5:1600-14.

52. Laugesen A, Hojfeldt JW, Helin K. Molecular Mechanisms Directing PRC2 Recruitment and H3K27 Methylation. Mol Cell 2019;74:8-18.

53. Huang X, Yan J, Zhang M, et al. Targeting Epigenetic Crosstalk as a Therapeutic Strategy for EZH2-Aberrant Solid Tumors. Cell 2018;175:186-199.e19.

54. Li H, Zhang R. Role of EZH2 in Epithelial Ovarian Cancer: From Biological Insights to Therapeutic Target. Front Oncol 2013;3:47.

55. Bartel DP. MicroRNAs: genomics, biogenesis, mechanism, and function. Cell 2004;116:281-97.

56. Bartel DP. MicroRNAs: target recognition and regulatory functions. Cell 2009;136:215-33.

57. Bartels CL, Tsongalis GJ. MicroRNAs: novel biomarkers for human cancer. Clin Chem 2009;55:623-31.

58. Sato F, Tsuchiya S, Meltzer SJ, et al. MicroRNAs and epigenetics. FEBS J 2011;278:1598-609.

59. Ramassone A, Pagotto S, Veronese A, et al. Epigenetics and MicroRNAs in Cancer. Int J Mol Sci 2018;19:459. 
60. Wu Y, Strawn E, Basir Z, et al. Aberrant expression of deoxyribonucleic acid methyltransferases DNMT1, DNMT3A, and DNMT3B in women with endometriosis. Fertil Steril 2007;87:24-32.

61. Xue Q, Lin Z, Cheng YH, et al. Promoter methylation regulates estrogen receptor 2 in human endometrium and endometriosis. Biol Reprod 2007;77:681-7.

62. Rocha-Junior CV, Da Broi MG, Miranda-Furtado CL, et al. Progesterone Receptor B (PGR-B) Is Partially Methylated in Eutopic Endometrium From Infertile Women With Endometriosis. Reprod Sci 2019;26:1568-74.

63. Noël JC, Anaf V, Borghese B, et al. The steroidogenic factor-1 protein is not expressed in various forms of endometriosis but is strongly present in ovarian cortical or medullary mesenchymatous cells adjacent to endometriotic foci. Fertil Steril 2011;95:2655-7.

64. Matsuzaki S, Darcha C, Maleysson E, et al. Impaired down-regulation of $\mathrm{E}$-cadherin and beta-catenin protein expression in endometrial epithelial cells in the mid-secretory endometrium of infertile patients with endometriosis. J Clin Endocrinol Metab 2010;95:3437-45.

65. Wang M, Hao C, Huang X, et al. Aberrant Expression of lncRNA ( HOXA11-AS1) and Homeobox A ( HOXA9, HOXA10, HOXA11, and HOXA13) Genes in Infertile Women With Endometriosis. Reprod Sci 2018;25:654-61.

66. Zheng J, Luo X, Bao J, et al. Decreased Expression of HOXA10 May Activate the Autophagic Process in Ovarian Endometriosis. Reprod Sci 2018;25:1446-54.

67. Wright K. Santanam N. The epigenetic role of EZH2FOXP3 crosstalk in endometriosis and its associated pain. Fertility and Sterility 2015;104:e165.

68. Colon-Caraballo M, Torres-Reveron A, Soto-Vargas JL, et al. Effects of histone methyltransferase inhibition in endometriosis. Biol Reprod 2018;99:293-307.

69. Zhang Q, Dong P, Liu X, et al. Enhancer of Zeste homolog 2 (EZH2) induces epithelial-mesenchymal transition in endometriosis. Sci Rep 2017;7:6804.

70. Monteiro JB, Colon-Diaz M, Garcia M, et al. Endometriosis is characterized by a distinct pattern of histone 3 and histone 4 lysine modifications. Reprod Sci 2014;21:305-18.

71. Bjorkman S, Taylor HS. MicroRNAs in endometriosis: biological function and emerging biomarker candidatesdagger. Biol Reprod 2019;100:1135-46.

72. Pan Q, Luo X, Toloubeydokhti T, et al. The expression profile of micro-RNA in endometrium and endometriosis and the influence of ovarian steroids on their expression. Mol Hum Reprod 2007;13:797-806.
73. Wang S, Zhang M, Zhang T, et al. microRNA-141 inhibits TGF-beta1-induced epithelial-to-mesenchymal transition through inhibition of the TGF-beta1/SMAD2 signalling pathway in endometriosis. Arch Gynecol Obstet 2020;301:707-14.

74. Gloss BS, Samimi G. Epigenetic biomarkers in epithelial ovarian cancer. Cancer Lett 2014;342:257-63.

75. Bernards SS, Pennington KP, Harrell MI, et al. Clinical characteristics and outcomes of patients with BRCA1 or RAD51C methylated versus mutated ovarian carcinoma. Gynecol Oncol 2018;148:281-5.

76. Wu Q, Lothe RA, Ahlquist T, et al. DNA methylation profiling of ovarian carcinomas and their in vitro models identifies HOXA9, HOXB5, SCGB3A1, and CRABP1 as novel targets. Mol Cancer 2007;6:45.

77. Widschwendter M, Apostolidou S, Jones AA, et al. HOXA methylation in normal endometrium from premenopausal women is associated with the presence of ovarian cancer: a proof of principle study. Int J Cancer 2009;125:2214-8.

78. Fu LJ, Zhang SL. Expression of RASSF1A in epithelial ovarian cancers. Eur Rev Med Pharmacol Sci 2015;19:813-7.

79. Beckedorff FC, Ayupe AC, Crocci-Souza R, et al. The intronic long noncoding RNA ANRASSF1 recruits PRC2 to the RASSF1A promoter, reducing the expression of RASSF1A and increasing cell proliferation. PLoS Genet 2013;9:e1003705.

80. Chmelarova M, Krepinska E, Spacek J, et al. Methylation in the p53 promoter in epithelial ovarian cancer. Clin Transl Oncol 2013;15:160-3.

81. Mvunta DH, Miyamoto T, Asaka R, et al. SIRT1 Regulates the Chemoresistance and Invasiveness of Ovarian Carcinoma Cells. Transl Oncol 2017;10:621-31.

82. Wang X, Wang H, Xu B, et al. Depletion of H3K79 methyltransferase Dot1L promotes cell invasion and cancer stem-like cell property in ovarian cancer. Am J Transl Res 2019;11:1145-53.

83. Chan MW, Huang YW, Hartman-Frey C, et al. Aberrant transforming growth factor beta1 signaling and SMAD4 nuclear translocation confer epigenetic repression of ADAM19 in ovarian cancer. Neoplasia 2008;10:908-19.

84. Liu J, Chen W, Zhang H, et al. miR-214 targets the PTEN-mediated PI3K/Akt signaling pathway and regulates cell proliferation and apoptosis in ovarian cancer. Oncol Lett 2017;14:5711-8.

85. Vecchione A, Belletti B, Lovat F, et al. A microRNA signature defines chemoresistance in ovarian cancer through modulation of angiogenesis. Proc Natl Acad Sci U S A 2013;110:9845-50. 
86. Bitler BG, Aird KM, Zhang R. Epigenetic synthetic lethality in ovarian clear cell carcinoma: EZH2 and ARID1A mutations. Mol Cell Oncol 2015;3:e1032476.

87. Samartzis EP, Samartzis N, Noske A, et al. Loss of ARID1A/BAF250a-expression in endometriosis: a biomarker for risk of carcinogenic transformation? Mod Pathol 2012;25:885-92.

88. Wiegand KC, Shah SP, Al-Agha OM, et al. ARID1A mutations in endometriosis-associated ovarian carcinomas. N Engl J Med 2010;363:1532-43.

89. Ren F, Wang DB, Li T, et al. Identification of differentially methylated genes in the malignant transformation of ovarian endometriosis. J Ovarian Res 2014;7:73.

90. Dinulescu DM, Ince TA, Quade BJ, et al. Role of K-ras and Pten in the development of mouse models of endometriosis and endometrioid ovarian cancer. Nat Med 2005;11:63-70.

91. Lv J, Zhu Q, Jia X, et al. In Vitro and In Vivo Effects of Tumor Suppressor Gene PTEN on Endometriosis: An Experimental Study. Med Sci Monit 2016;22:3727-36.

92. Woenckhaus J, Steger K, Sturm K, et al. Prognostic value of PIK3CA and phosphorylated AKT expression in ovarian cancer. Virchows Arch 2007;450:387-95.

93. Takei Y, Saga Y, Mizukami H, et al. Overexpression of PTEN in ovarian cancer cells suppresses i.p. dissemination and extends survival in mice. Mol Cancer Ther 2008;7:704-11.

94. Colon-Caraballo M, Soto-Vargas J, Urrutia R, et al. Dissecting the role of $\mathrm{EZH} 2$ and $\mathrm{H} 3 \mathrm{~K} 27 \mathrm{me} 3$ in endometriosis The FASEB Journal 2017;31:Supplement 1058.1.

95. Chen Y, Du H, Bao L, et al. LncRNA PVT1 promotes ovarian cancer progression by silencing miR-214. Cancer Biol Med 2018;15:238-50.

96. Braicu OL, Budisan L, Buiga R, et al. miRNA expression profiling in formalin-fixed paraffin-embedded endometriosis and ovarian cancer samples. Onco Targets Ther 2017;10:4225-38.

97. Jiang J, Zhang Y, Yu C, et al. MicroRNA-492 expression promotes the progression of hepatic cancer by targeting PTEN. Cancer Cell Int 2014;14:95.

98. Li H, Huang W, Luo R. The microRNA-325 inhibits hepatocellular carcinoma progression by targeting high mobility group box 1. Diagn Pathol 2015;10:117.

99. Chan JK, Blansit K, Kiet T, et al. The inhibition of miR21 promotes apoptosis and chemosensitivity in ovarian cancer. Gynecol Oncol 2014;132:739-44.

100.Wang WT, Zhao YN, Han BW, et al. Circulating
microRNAs identified in a genome-wide serum microRNA expression analysis as noninvasive biomarkers for endometriosis. J Clin Endocrinol Metab 2013;98:281-9.

101.Feng X, Liu N, Deng S, et al. miR-199a modulates cisplatin resistance in ovarian cancer by targeting Hiflalpha. Onco Targets Ther 2017;10:5899-906.

102.Petracco R, Grechukhina O, Popkhadze S, et al. MicroRNA 135 regulates HOXA10 expression in endometriosis. J Clin Endocrinol Metab 2011;96:E1925-33.

103.Petracco R, Dias ACO, Taylor H, et al. Evaluation of miR$135 \mathrm{a} / \mathrm{b}$ expression in endometriosis lesions. Biomed Rep 2019;11:181-7.

104. Tang W, Jiang Y, Mu X, et al. MiR-135a functions as a tumor suppressor in epithelial ovarian cancer and regulates HOXA10 expression. Cell Signal 2014;26:1420-6.

105.Zhang Y, Yan J, Pan X. miR-141-3p affects apoptosis and migration of endometrial stromal cells by targeting KLF12. Pflugers Arch 2019;471:1055-63.

106. Mak CS, Yung MM, Hui LM, et al. MicroRNA-141 enhances anoikis resistance in metastatic progression of ovarian cancer through targeting KLF12/Sp1/survivin axis. Mol Cancer 2017;16:11.

107. Wu RL, Ali S, Bandyopadhyay S, et al. Comparative Analysis of Differentially Expressed miRNAs and their Downstream mRNAs in Ovarian Cancer and its Associated Endometriosis. J Cancer Sci Ther 2015;7:258-65.

108. Nikpayam E, Tasharrofi B, Sarrafzadeh S, et al. The Role of Long Non-Coding RNAs in Ovarian Cancer. Iran Biomed J 2017;21:3-15.

109. Cui D, Ma J, Liu Y, et al. Analysis of long non-coding RNA expression profiles using RNA sequencing in ovarian endometriosis. Gene 2018;673:140-8.

110. Wang Y, Li Y, Yang Z, et al. Genome-Wide Microarray Analysis of Long Non-Coding RNAs in Eutopic Secretory Endometrium with Endometriosis. Cell Physiol Biochem 2015;37:2231-45.

111. Kung JT, Colognori D, Lee JT. Long noncoding RNAs: past, present, and future. Genetics 2013;193:651-69.

112.Zou A, Liu R, Wu X. Long non-coding RNA MALAT1 is up-regulated in ovarian cancer tissue and promotes SK-OV-3 cell proliferation and invasion. Neoplasma 2016;63:865-72.

113. Liu H, Zhang Z, Xiong W, et al. Long non-coding RNA MALAT1 mediates hypoxia-induced pro-survival autophagy of endometrial stromal cells in endometriosis. J Cell Mol Med 2019;23:439-52.

114.Ivanga M, Labrie Y, Calvo E, et al. Temporal analysis 
of E2 transcriptional induction of PTP and MKP and downregulation of IGF-I pathway key components in the mouse uterus. Physiol Genomics 2007;29:13-23.

115. Ghazal S, McKinnon B, Zhou J, et al. H19 lncRNA alters stromal cell growth via IGF signaling in the endometrium of women with endometriosis. EMBO Mol Med 2015;7:996-1003.

116.Zheng ZG, Xu H, Suo SS, et al. The Essential Role of H19 Contributing to Cisplatin Resistance by Regulating Glutathione Metabolism in High-Grade Serous Ovarian Cancer. Sci Rep 2016;6:26093.

117.He A, Liu Y, Chen Z, et al. Over-expression of long noncoding RNA BANCR inhibits malignant phenotypes of human bladder cancer. J Exp Clin Cancer Res 2016;35:125.

118.Zhu MB, Chen LP, Hu M, et al. Effects of lncRNA BANCR on endometriosis through ERK/MAPK pathway. Eur Rev Med Pharmacol Sci 2019;23:6806-12.

119.Xu X, Pan L, Zhuo M, et al. Increased expression of LncRNA BANCR and its prognostic significance in human epithelial ovarian cancer. Eur J Gynaecol Oncol 2017;38:449-52.

120. Yang Q, Yang Y, Zhou N, et al. Epigenetics in ovarian cancer: premise, properties, and perspectives. Mol Cancer 2018;17:109.

121. Muscolini M, Cianfrocca R, Sajeva A, et al. Trichostatin A up-regulates p73 and induces Bax-dependent apoptosis in cisplatin-resistant ovarian cancer cells. Mol Cancer Ther 2008;7:1410-9.

122.Ding YB, Long CL, Liu XQ, et al. 5-aza-2'-deoxycytidine leads to reduced embryo implantation and reduced expression of DNA methyltransferases and essential endometrial genes. PLoS One 2012; 7:e45364.

123. Matei D, Fang F, Shen C, et al. Epigenetic resensitization to platinum in ovarian cancer. Cancer Res 2012;72:2197-205.

124. Strauss J, Figg WD. Using Epigenetic Therapy to Overcome Chemotherapy Resistance. Anticancer Res

Cite this article as: Brunty S, Mitchell B, Bou-Zgheib N, Santanam N. Endometriosis and ovarian cancer risk, an epigenetic connection. Ann Transl Med 2020;8(24):1715. doi: 10.21037/atm-20-2449
2016;36:1-4.

125.Marchetti C, Muzii L, Romito A, et al. First-line treatment of women with advanced ovarian cancer: focus on bevacizumab. Onco Targets Ther 2019;12:1095-103.

126. Stone ML, Chiappinelli KB, Li H, et al. Epigenetic therapy activates type I interferon signaling in murine ovarian cancer to reduce immunosuppression and tumor burden. Proc Natl Acad Sci U S A 2017;114:E10981-E10990.

127. Miranda TB, Cortez CC, Yoo CB, et al. DZNep is a global histone methylation inhibitor that reactivates developmental genes not silenced by DNA methylation. Mol Cancer Ther 2009;8:1579-88.

128. Bratkowski M, Yang X, Liu X. An Evolutionarily Conserved Structural Platform for PRC2 Inhibition by a Class of Ezh2 Inhibitors. Sci Rep 2018;8:9092.

129. Oki S, Sone K, Oda K, et al. Oncogenic histone methyltransferase EZH2: A novel prognostic marker with therapeutic potential in endometrial cancer. Oncotarget 2017;8:40402-11.

130. Karakashev S, Zhu H, Wu S, et al. CARM1-expressing ovarian cancer depends on the histone methyltransferase EZH2 activity. Nat Commun 2018;9:631.

131. Seo SK, Lee JH, Chon SJ, et al. Trichostatin A Induces NAG-1 Expression and Apoptosis in Human Endometriotic Stromal Cells. Reprod Sci 2018;25:1349-56.

132.Lu Y, Nie J, Liu X, et al. Trichostatin A, a histone deacetylase inhibitor, reduces lesion growth and hyperalgesia in experimentally induced endometriosis in mice. Hum Reprod 2010;25:1014-25.

133. Wu Y, Guo SW. Histone deacetylase inhibitors trichostatin A and valproic acid induce cell cycle arrest and p21 expression in immortalized human endometrial stromal cells. Eur J Obstet Gynecol Reprod Biol 2008;137:198-203.

134.Liu M, Liu X, Zhang Y, et al. Valproic acid and progestin inhibit lesion growth and reduce hyperalgesia in experimentally induced endometriosis in rats. Reprod Sci 2012;19:360-73. 